The issue of "access" is central to epidemiological research. Once a set of "interesting" data has been targeted, the researcher must overcome the ethical, legal and other conditions to actually access the data and samples of interest. In addition, study specific conditions (eg, access to aggregate data only) may limit the choice of methodologies to analyse synthesizable data.

A more "open access" culture for research data requires properly managing and accessing the massive amount of potentially sensitive information in a way that will be of optimal use for the scientific community while also ensuring proper protection and respect of participants (including their privacy and confidentiality). The "law" is often blamed for creating "undue hurdles" to international access to research data, but are we focusing on the real problem?

We will discuss some ethical, legal and social issues challenges faced by researchers who wish to access multiple research infrastructures to conduct epidemiological research. We also identify avenues of innovative solutions being proposed to meet some of these challenges.

\section{3-5.6 STANDARDISATION OF CLINICAL AND PERSONAL CHARACTERISTICS USING INTERNATIONAL STANDARD NOMENCLATURE AND ICT SOLUTIONS}

doi:10.1136/jech.2011.142976b.6

\section{J Mintzer. ${ }^{*}$ Coriell Institute of Medical Research, New York, USA}

The compilation, retrieval, use, storage, and distribution of genotypic and phenotypic data associated with the collection, storage, processing, and distribution of biomaterials managed by biobanks is insatiable. Clinical trials, observational studies, and fundamental basic science research is "pre-qualifying" biomaterials sought and used in research based on the quality of data submitted with a biomaterial as well as the quantifiable data associated with its processing. Additionally, longitudinal designs to study the course of disease may require multiple specimen submissions tied with its time specific associated phenotypic data. Furthermore, significant attention is being given to defining "clinical data elements" or CDE's to assure that data with the most significant clinical relevance are collected at each time interval. Clearly, it is no trivial task to define a $\mathrm{CDE}$ and correlate its potential relevance to the research enterprise. This presentation will discuss how said data are collected, evaluated for its relevance (power), and how it is made available to the scientific community using the Coriell Personalised Medicine Collaborative and Coriell's biobanking models as case presentations.

\subsection{EARLY CAREER RESEARCHERS SESSION}

\section{3-6.1 PUTTING EMERGING EPIDEMIOLOGIST'S VOICES ON THE}

doi:10.1136/jech.2011.142976b.7

\begin{abstract}
${ }^{1} \mathrm{~S}$ Abdel-Maqsoud, ${ }^{2} \mathrm{~N}$ Brewer, ${ }^{*} \mathrm{M} \mathrm{C}$ Restrepo, ${ }^{*}{ }^{4}$ E Villalonga Olives. ${ }^{*}$ Alexandria University Students' Clinic and Hospital, Alexandria University, Alexandria, Egypt; ${ }^{2}$ Centre for Public Health Research, Massey University, Wellington, New Zealand; ${ }^{3}$ Federal University of Pelotas, Pelotas, Brazil; ${ }^{4}$ Mateu Orfila research center, Public Health Agency of Barcelona, IMIM-Parc de Salut Mar, Barcelona Biomedical Research Park
\end{abstract}

This Early Career Researcher (ECR) session will be the first such session at an IEA World Congress of Epidemiology. The session is being held in order to build on the work of an ad hoc ECR Committee which has prepared a report to the IEA Council recommending the creation of a formal IEA ECR group. The session will report on what the ad hoc committee has been doing, and get feedback and suggestions on how to proceed, as well as have a discussion with Professor Cesar Victora and Professor Shah Ebrahim about how to make health research work towards development. It is hoped, and intended, that the incoming
IEA Council will then formally establish an ECR group within IEA. This group would aim to develop a network of emerging epidemiologists to enhance global scientific collaboration. We look forward to the active participation of early career epidemiologists from across the world in this session as it will be an extraordinary opportunity to establish a global dialogue among early career health professionals engaged in research and teaching of epidemiology.

\section{Wednesday 10 August 2011 Parallel session 4 4.1 SPECIFIC CHALLENGES TO GLOBAL HEALTH}

\section{Chair: Dr. Vinod K Srivastava, India 04-1.1 NEONATAL CONDITIONS AND AUTISM SPECTRUM DISORDERS}

doi:10.1136/jech.2011.142976b.8

${ }^{1} \mathrm{H} O$ Atladottir, ${ }^{2} \mathrm{~T}$ B Henriksen, ${ }^{3} \mathrm{M}$ B Lauritsen, ${ }^{4} \mathrm{E} T$ Parner. ${ }^{1}$ Department of Epidemiology, School of Public Health, University of Aarhus, Aarhus, Denmark; ${ }^{2}$ Perinatal Research Unit, Department of Pediatrics, Aarhus University Hospital, Aarhus, Denmark; ${ }^{3}$ Regional Centre for Child and Adolescent Psychiatry, Aarhus University Hospital, Aarhus, Denmark; ${ }^{4}$ Department of Biostatistics, School of Public Health, University of Aarhus, Aarhus, Denmark

Background Autism spectrum disorders (ASDs) are disorders of neural development characterised by impaired social interaction and communication, and by restricted and repetitive behaviour. Only few previous studies have investigated neonatal conditions and the risk for ASDs.

Objectives To use Danish population based sample and register based information to investigate whether neonatal conditions are associated to the later development of ASD.

Methods A Danish population based cohort study, including all singletons born in Denmark from 1994, through 2005, a total of 581493 children. Data were retrieved from the Danish National Hospital Register and the Danish Psychiatric Central Register. Data were analysed using Cox proportional hazards regression. All analyses were stratified by gestational age (term vs preterm birth).

Results A total of 4846 children were diagnosed with ASD during the follow-up time. We found an increased risk of ASD after exposure to a variety of neonatal conditions. For children born at term, we found an increased risk of ASD after perinatal hypoxia: HR 5.0 (95\% CI 2.1 to 11.9), neonatal seizures: 2.2 (1.4 to 3.5), intracranial haemorrhage: HR 3.0 (1.4 to 6.2), neonatal hypoglycemia: HR 1.5 (1.3 to 1.8), and neonatal septicaemia or meningitis: HR 1.8 (1.5 to 2.2). The results for children born preterm were similar as for children born at term.

Conclusions Different neonatal conditions are likely to cause neurological damage and alter brain development, and hence increase the risk of ASDs. This effect seems to be mediated through different pathways including lack of oxygen, glucose, and possibly through activated immune function during early neonatal life.

\section{4-1.2 IDENTIFYING AN OPTIMAL EXPOSURE METRIC FOR MEASURING THE SHORT-TERM EFFECTS OF LOW INDOOR TEMPERATURES ON ASTHMATIC CHILDREN'S LUNG FUNCTION}

doi:10.1136/jech.2011.142976b.9

\begin{abstract}
${ }^{1} \mathrm{~N}$ Pierse, ${ }^{*}{ }^{2} \mathrm{R}$ Arnold, ${ }^{1} \mathrm{M}$ Keall, ${ }^{1} \mathrm{~J}$ Crane, ${ }^{1} \mathrm{P}$ Howden-Chapman, ${ }^{3} \mathrm{M}$ Cunningham. ${ }^{1}$ University of Otago, Wellington, New Zealand; ${ }^{2}$ Victoria University, Wellington, New Zealand; ${ }^{3}$ BRANZ, Porirua, New Zealand
\end{abstract}

Introduction Many epidemiological studies have shown that low outdoor temperatures lead to increased mortality and 
hospitalisation, generally finding the strongest association to be with temperature below a given threshold measured at a lag of 7-14 days. In contrast, very little work has been done on the health effects of exposure to low indoor temperatures, and none on the best metric of this exposure, despite the fact that people have greater exposure to the indoor environment. The scarcity of studies on the association between indoor temperature and health is due to the difficulty in measuring indoor temperatures and health outcomes regularly and simultaneously over an extended time period.

Methods The Heating Housing and Health Study is an RCT which investigates the effect of installing heaters in asthmatic children's homes. It has detailed measurements of lung function (daily) and indoor temperature (hourly).

Lung function and indoor temperature were measured for 309 children over 12049 child-days. For four measures of lung function (PEFR morning, PEFR evening, FEV1 morning and FEV1 evening) we attempted to find the strongest association between exposure to low temperatures below particular thresholds averaged over various periods.

Results Indoor temperatures were found to have a small, but significant, association with short-term variations in the lung function in children with asthma. This association was greatest for temperatures below $11^{\circ} \mathrm{C}$ in the child's bedroom averaged over the preceding 11 days.

Conclusion These findings provide valuable information for future studies looking at the effects of low indoor temperatures on respiratory health.

\section{4-1.3 OVERWEIGHT IN SHORT AND TALL CHILDREN}

doi:10.1136/jech.2011.142976b.10

${ }^{1} \mathrm{P}$ van Dommelen, ${ }^{*}{ }^{2} \mathrm{M}$ L A de Kroon, ${ }^{1} Y$ Schönbeck, ${ }^{1,3} \mathrm{~S}$ van Buuren. ${ }^{1}$ Netherlands Organization for Applied Scientific Research, Leiden, The Netherlands; ${ }^{2} \mathrm{VU}$ University Medical Center, Amsterdam, The Netherlands; ${ }^{3}$ Utrecht University, Utrecht, The Netherlands

Introduction It is known that body mass index is correlated with height. This suggests that the prevalence rates of overweight and obesity differ between tall and short children. Our aim is to study the impact of height during childhood on the national prevalence rates of overweight and obesity at childhood and adulthood.

Methods We used cross-sectional growth data from the previous two Dutch nationwide surveys performed in $1997(\mathrm{n}=14500)$ and 2009 ( $n=10129)$ and longitudinal data from birth to $18 \mathrm{y}(\mathrm{n}=708)$. We selected children from 2 to $10 \mathrm{y}$ (before onset of puberty) from the two surveys. Overweight (including obesity) and obesity were calculated by cut-offs for body mass index $\left(\mathrm{kg} / \mathrm{m}^{2}\right)$ according to International Obesity Task Force. Short was defined as $<-1 \mathrm{SD}$ and tall by $>+1 \mathrm{SD}$.

Results In 1997, the prevalence rates of childhood overweight and obesity were respectively $5.1 \%$ and $0.8 \%$ in short children, and $17.0 \%$ and $3.0 \%$ in tall children. In 2009 , these figures were $6.9 \%$ and $1.1 \%$ in short children, and $22.9 \%$ and $5.5 \%$ in tall children. The RRs of childhood overweight and obesity of tall vs short children were respectively 3.3 (95\% CI 2.6 to 4.2 ) and 4.4 (95\% CI 2.4 to 7.9 ). However, these RRs were lower at adulthood, respectively $1.3(95 \%$ CI 0.5 to 3.7 ) and 1.5 (0.98 to 2.2).

Conclusion There is a height bias in the prevalence of overweight and obesity. Correcting for height gives better insight into (inter) national comparisons and secular trends of overweight and obesity at childhood. We will present the figures and methodological explanation of the height bias.

\section{4-1.4 NATIONAL INCOME AND INCOME INEQUALITY, FAMILY AFFLUENCE AND LIFE SATISFACTION AMONG ADOLESCENTS IN 35 COUNTRIES}

doi:10.1136/jech.2011.142976b.11

${ }^{1} \mathrm{~K}$ Levin, ${ }^{*} \mathrm{~T}$ Torsheim, ${ }^{3} \mathrm{~W}$ Vollebergh, ${ }^{4} \mathrm{M}$ Richter, ${ }^{5} \mathrm{C}$ Davies, ${ }^{6} \mathrm{C}$ Schnohr, ${ }^{6} \mathrm{P}$ Due, ${ }^{1} \mathrm{C}$ Currie. 'University of Edinburgh, Edinburgh, UK; ${ }^{2}$ University of Bergen, Bergen, Norway; ${ }^{3}$ University of Utrecht, Utrecht, The Netherlands; ${ }^{4}$ University of Bielefeld, Bielefeld, Germany; ${ }^{5}$ University of Glasgow, Glasgow, UK; ${ }^{6}$ University of Southern Denmark, Copenhagen, Denmark

Introduction This study examines 13 year olds' life satisfaction crossnationally and investigates variation in its relationship with family affluence, and the impact of national income and income inequality on this relationship.

Methods Data from the 2006 Health Behaviour in School-aged Children: WHO-collaborative Study ( $N=58352$ across 35 countries) were analysed using multilevel linear and logistic regression for outcome measures life satisfaction score and binary high / low life satisfaction.

Results National income and income inequality were associated with national mean life satisfaction score and prevalence of high life satisfaction. The relationship between life satisfaction and family affluence was curvilinear and varied cross-nationally, for example, family affluence was not related to life satisfaction in Denmark and France, while steep relationships were seen in England, Lithuania, Macedonia, Turkey and Romania. When the data were modelled simultaneously, GDP (PPP US\$) and Gini were not in themselves associated with the life satisfaction, however this relationship varied depending on young people's relative affluence. Socioeconomic inequalities were greatest- steepest gradients were seen- in poorer countries (lower GDP (PPP US\$) and in countries with unequal income distribution (higher Gini score).

Conclusions The data were collected prior to the global economic recession and therefore this study may have underestimated current socioeconomic inequalities in life satisfaction and international variation in inequalities. As adolescence is a critical period where many patterns of health and health behaviour are formed, this study highlights the importance of monitoring cross-national inequalities and identifying and addressing national mediating factors during this life stage.

\section{4-1.5 MACRO-LEVEL DETERMINANTS OF HEALTH AMONG ADOLESCENTS IN WELFARE STATES: A MULTI-LEVEL ANALYSIS}

doi:10.1136/jech.2011.142976b.12

${ }^{1} \mathrm{~K}$ Rathmann, ${ }^{*} \mathrm{M}$ Richter. ${ }^{1}$ Berlin Graduate School of Social Sciences, Humboldt Universität zu Berlin, Berlin, Germany; ${ }^{2}$ Institute of Social and Preventive Medicine, University of Bern, Bern, Switzerland

Introduction Previous studies have shown that macro-level factors, such as income inequality, wealth of countries or welfare state characteristics are crucial determinants of health and health inequalities among adults. So far, comparative research has rarely focused on children and adolescents. This study is among the first to examine whether different macro-level determinants are associated with differences in subjective health and socio-economic related health inequalities among adolescents in high-developed welfare states.

Methods Data were collected from the school based "Health Behaviour in School-aged Children"-study in 2005/2006, which includes 11- to 15-year old students from 27 European and North American countries $(\mathrm{N}=134.632)$, classified into five regimes (Scandinavian, Bismarckian, Liberal, Southern, Eastern). Hierarchical linear regression models were conducted for multiple complaints in health, including stepwise individual (family affluence) and 